\title{
Feasibility of incorporating a bed-rest exercise program during a twin pregnancy: Case reports
}

\author{
Chantale R. Brun ${ }^{1 \#}$, Jo-Anne Hammond ${ }^{2,3,4}$, Michelle F. Mottola ${ }^{2,5,6}$ \\ ${ }^{1}$ School of Kinesiology and Recreation, Université de Moncton, Moncton, Canada \\ ${ }^{2}$ Samuel McLaughlin Foundation-Exercise \& Pregnancy Laboratory, London, Canada \\ ${ }^{3}$ Department of Family Medicine, Schulich School of Medicine \& Dentistry, University of Western Ontario, London, Canada \\ ${ }^{4}$ St. Joseph's Health Care, London, Canada \\ ${ }^{5}$ School of Kinesiology, Faculty of Health Sciences, University of Western Ontario, London, Canada \\ ${ }^{6}$ Department of Anatomy \& Cell Biology, Schulich School of Medicine \& Dentistry, University of Western Ontario, London, Canada \\ Email: " chantale.brun@umoncton.ca
}

Received 7 May 2012; revised 10 June 2012; accepted 22 June 2012

\begin{abstract}
Considering the maternal risk for both short- and long-term disability imposed by activity restriction, it is of great concern that the antepartum symptoms of bed rest are still evident in the postpartum period. The benefits of bed-rest-exercise may alleviate or even prevent physiological distresses or deconditioning associated with activity restriction, thus, eliminating or stabilizing, factors that may augment existing complications. This is the first study to investigate the feasibility of incorporating a bed-rest exercise program in a specific hospitalized twin pregnancy case. The maternal physiological responses of heart rate, blood pressure and uterine contractions to a four week bed-rest resistance program were measured in a woman hospitalized due to complications during her twin pregnancy. A second participant (same diagnoses) was included as a control. The bedrest-exercise reduced the degree of swelling in the lower extremities, promoted more energy during the day and a better night's sleep. There were no significant differences in uterine contractions pre vs post protocol and all babies were born healthy.
\end{abstract}

Keywords: Exercise; Bed Rest; Pregnancy; Antepartum; Muscle Conditioning; Training

\section{INTRODUCTION}

Bed-rest has been extensively researched, in the nonpregnant population, especially by the National Aeronautics and Space Administration (NASA) and other aerospace scientists who are interested in the effects of microgravity on human physiology. Bed rest is also one of

\footnotetext{
*Supported by Child Health Research Institute, London, Canada.

${ }^{*}$ Corresponding author.
}

the oldest treatments in health care and therefore not an uncommon treatment for patients with chronic disease or injury as well as to treat women with complications of pregnancy.

Prolonged bed-rest has been associated with decreases in cardiovascular and muscular performance with reduced physical work capacity [1-7]. An active individual who is suddenly restricted from daily activities may, therefore, experience several physiological side-effects related to bed-rest (or activity restriction). Patients with chronic disease or injury are prescribed bed-rest routinely to improve the disease state or to help with the healing process. Thus, the side effects of activity restriction should be distinguished from the disease process for which the individual was bed-rested and the resulting side effects of activity restriction.

Despite non-existent scientific evidence to show efficacy, bed-rest is also prescribed as the primary mode of care for high-risk pregnant women [8,9]. Limited research evidence would suggest that pregnant women are also affected by the physiological effects of activity restriction. Important antepartum side effects range from loss of muscle strength and muscle atrophy, weight loss, dizziness, headaches and indigestion to possible lifethreatening thromboembolic events [10-15]. Bed rest may therefore be responsible for complicating the antepartum period, regardless of the disease process for which bed rest was originally prescribed.

When considering the maternal risk for both short- and long-term disability imposed by bed rest as well as the lack of attention given to recovery (or rehabilitation), it is concerning that the antepartum symptoms of bed rest are still evident in the postpartum period. For instance, during the first week postpartum, women on complete and partial bed-rest complained of muscle soreness to the lower back, upper and lower leg, arms and heels [11,12]. They also reported mobility problems such as difficulty 
with descending and ascending stairs, knees buckling, needing support to walk or sit [11]. Symptoms such as fatigue, headache, mood changes, tenseness, difficulty concentrating, back muscle soreness, and dry skin were still present at six weeks postpartum [16]. The presence of such symptoms is proof for the physiological deconditioning caused by the antenatal bed rest prescription as well as for the prolonged recovery in the postpartum period. Yet they seem to go unnoticed. Interventions should exist at both levels (antepartum and postpartum) especially when healthy postpartum women show a high prevalence of morbidity [17-19]. Recovery symptoms such as exhaustion/extreme tiredness and backache are still apparent six months after childbirth (81\% of women reported health problems) [18]. Bed rest may therefore be responsible for not only complicating the antepartum period but also the postpartum period, regardless of the disease process for which bed rest was originally prescribed, and the quality of life of both mother and family.

The benefits of bed-rest-exercise may alleviate or even prevent physiological distresses or deconditioning associated with activity restriction, thus, eliminating or stabilizing, factors that may augment existing complications. One must consider the benefits of exercise, the benefits of exercise during pregnancy, the possible detrimental effects of activity restriction, and the feasibility of exercising in a hospital setting before incorporating a bedrest-exercise intervention program for activity restricted antenatal women. The benefits of incorporating a bedrest-exercise program have yet to be fully investigated in the antepartum hospitalized population, but are well documented in the non pregnant population [20-24]. The first study was conducted in 1992, by Mayberry and colleges, in order to assess the short-term effects of controlled bed-rested exercise on uterine activity of women diagnosed with preterm labour [25]. Preliminary findings suggested that women diagnosed with preterm labour may be able to participate safely in an exercise program with minimal risk for increased uterine activity after bedrest exercise. Twenty years after this first study, our lab has investigated the effects of a bed-rest-exercise session on hospitalized antenatal women. Results showed that women respond well to one bout of a bed-rest session of resistance training when examining heart rate, blood pressure and uterine contractions [26]. In the current study, the primary objective was to implement and monitor a four week bed-rest resistance training program (while listening to music) in a patient hospitalized due to complications during her twin pregnancy. A second patient (twin pregnancy) was also included in the study as a control. She listened to same music but did not exercise. To our knowledge this is the first pilot study investigating the effects of a bed-rest-exercise program in specific hospitalized twin pregnancy cases.

\section{CASE REPORTS}

Two hospitalised pregnant women, age and diagnosismatched, were randomly recruited from a larger study investigating the effects of acute bed-rest-exercise [26]. Both women had twin pregnancies, were non-smokers and bed-rested for at least 6 weeks. Study participants gave written informed consent, as approved by the human research board for Health Sciences at The University of Western Ontario. Both participants also received medical clearance from their health care provider in order to participate in the study. They were then interviewed for previous history of activity (prior to bed rest), amount of current activity restriction, medications, age, lifestyle status (e.g., smoking), and gestational age.

Characteristics of the two participants are presented in Table 1. Both were sedentary prior to bed-rest, with similar biological and gestational ages and the amount of time they were bed-rested prior to the study.

\subsection{Case 1}

The first case (experimental) consisted of an antepartum woman thirty years of age, hospitalized at 27 weeks gestational age (GA), due to a shortened cervix during her twin pregnancy. Her bed-rest prescription was to remain in the hospital bed at all times with the exception of bathroom privileges. Other than work around the house, she was not physically active. This was her third pregnancy, with two children ( 2 and 4 years of age) at home. Height and weight were $1.6 \mathrm{~m}$ and $109.2 \mathrm{~kg}$, respectively.

The bed-rest resistance program was implemented at 33 weeks gestational age, 2 - 3 sessions a week for four weeks. The protocol has been described previously [26]. Exercise sessions in weeks 2 and 3 were cancelled due to unexpected visitors coming in or simply feeling too tired on that particular day. She was induced at the end of

Table 1. Characteristics of the two study subjects.

\begin{tabular}{ccccc}
\hline & Maternal Age (years) & Gestational Age (wks) & Bed-Rest (wks) & Physical Activity (before bed-rest) \\
\hline Case 1 & 30 & 33 & 6 & House work no structured exercise \\
Case 2 & 34 & 28 & 8 & Walking not on regular basis \\
\hline
\end{tabular}

Case 1: bed-rest-exercise (with music) program; Case 2: bed-rest-control (music only). 
week 4 of the program and therefore only one exercise session was conducted during this particular week. Both babies were born healthy (Baby A: $2765 \mathrm{~g}$, APGAR 10 at 5 minutes; Baby B: $3265 \mathrm{~g}$, APGAR 10 at 5 minutes).

All exercise sessions were instructed and monitored as well as conducted at about the same time everyday (between 8:00 am and 9:45 am). The bed-rest muscle conditioning program consisted of upper and lower body exercises (biceps, triceps, rhomboids, calf and quadriceps muscle groups) using a dynaband (red resistance for upper body; green resistance for calf muscles). Quadriceps exercises were conducted without the dynaband. Since this type of exercise was completely new to the participant, fewer exercises were performed in the 30 minute sessions. The first week was therefore comprised of only upper body exercises and then gradually with time the lower body exercises were introduced.

Both HR and BP were somewhat high and inconsistent at rest (HR: ranges from 93 to $110 \mathrm{bpm}$; BP: ranges from $138 / 72$ to $124 / 80 \mathrm{mmHg}$ ). HR during exercise ranged from 120 - 134 beats per minute, and by $\mathrm{T}=50$ heart rate was lowered slightly but still higher than resting HR. In general, at twenty minutes post-exercise $(T=70 \mathrm{~min})$, $\mathrm{BP}$ and HR returned to resting values. In a few instances BP was lower 20 minutes post-exercise (130/80 vs $122 /$ $80 ; 138 / 72$ vs $130 / 72 ; 130 / 84$ vs $124 / 80$ ). There was only one uterine contraction monitored post bed-rest-exercise. She reported having felt a uterine contraction the day before starting the bed-rest-exercise program. No other uterine contractions were measured or reported before or after the bed-rest-exercise sessions for the remainder of the program.

After only one week on the exercise program, she reported having more energy during the day and sleeping better at night. She also noticed that the swelling in the lower extremities had diminished. The participant did keep a journal of her daily activities. Her daily activity ranged from sitting in bed, walking to the bathroom, doing her laundry and going down to the cafeteria (most times using a wheelchair).

\subsection{Case Report 2}

The second antepartum woman received no intervention and therefore acted as the control case. She was 34 years of age and was hospitalized due to a shortened cervix at 20 weeks GA during her twin pregnancy. This was her first pregnancy. Her bed-rest prescription was to stay in bed with bathroom privileges. She did engage in some exercise (mild walking) during her leisure time before and during her first trimester of pregnancy, but not always on a regular basis (approximately 2x/week for 30 minutes). C-1 was $1.63 \mathrm{~m}$ in height and weighed $64 \mathrm{~kg}$ at study entry.
The bed-rest control intervention was implemented at 28 weeks GA, three sessions a week for four weeks. Her 4 weeks data set is complete. She was able to participate in the study until delivery therefore her total time on the program was 6 weeks. However, for the purpose of this paper only the first 4 weeks have been reported. She went into labour at 33 weeks GA, had a vaginal delivery and two healthy babies (Baby A: 1890 g, APGAR 9 at 5 minutes; Baby B: $1825 \mathrm{~g}$, APGAR 9 at 5 minutes).

She listened to music for 30 minutes instead of conducting bed-rest-exercise. All sessions were supervised and monitored in the same manner as the bed-rest-exercise and conducted at about the same time of day (between 9:00 am and 9:45 am). The complete protocol has been described previously [26]. During most music sessions, she would fall asleep and had to be awakened in order to conduct post-intervention measures. She reported feeling relaxed at the end of each session. Two weeks into the program, she started to complain of swelling in her lower extremities, feeling very tired all the time, difficulty sleeping, no appetite and shortness of breath on exertion.

Resting maternal HR, BP and UC (pre-intervention) were monitored and recorded. All recorded parameters did not change dramatically from resting values during and after listening to music. In some instances HR was reduced slightly during or after the intervention (123 vs 111 bpm; 110 vs 108 bpm; 118 vs 109 bpm; 117 vs 104 bpm; respectively). However, by week 4, resting HR increased to $122 \mathrm{bpm}$ from study entry (115 bpm). Blood pressure was also a little higher at week 4 than at week 1 (108/62 vs 118/72 mmHg; respectively). There were no uterine contractions pre vs. post intervention.

She also kept a journal of her daily activities. Her daily activities ranged from reading, playing cards, weekly trips in wheelchair for ultrasounds, to occasional 2 minute walks to the kitchenette. She was very dedicated to following her bed-rest prescription.

\section{DISCUSSION}

Having conducted an acute bed-rest-exercise study previously in our lab [26], the primary goal of this study was to determine the feasibility and the implications of implementing a bed-rest-exercise program over several weeks in a woman hospitalized with a twin pregnancy. A bed-rest-exercise program was therefore applied to one patient on the antepartum hospital ward. Her results were then compared to a control woman with a similar activity history, gestational age and pregnancy. The key findings were no change in maternal BP or in the number of UC with the exercise intervention compared to the control case. These are case reports and their findings unfortunately cannot be generalized, however, they warrant 
analysis and discussion.

Treatment with activity restriction is based on the assumption that it is effective in preventing adverse pregnancy outcomes and that it is safe for both the woman and her fetus [27]. The physiological side-effects of bedrest on hospitalized antenatal women have been documented and have shown to be similar than those found for the non-pregnant population [11-14,28]. According to Bloomfield [1], the most important practical implications when working with bed-rested individuals, should be avoidance of prolonged immobility. When non-pregnant subjects were given an exercise bed-rest intervention program, they maintained heart and lung capacity as well as muscular integrity [1]. Therefore, if antenatal bed-rest complicates the postpartum period, bed-rest-exercise may help to alleviate or even prevent physiological distresses associated with activity restriction.

Both women were sedentary prior to and during their pregnancy and were both prescribed bed-rest with bathroom privileges. However, the women seemed to be a bit more active than what was prescribed. Case 1 made frequent visits in the hallway, laundry, cafeteria, and gift shop, sometimes in a wheel chair, sometimes not. When in bed these women were usually in a semi-recumbent position, however, sometimes they would simply sit up in bed or sit in a rocking chair. It would seem that activity is restricted but, they are not lying down in bed all day. Activity restriction may therefore better define the situation of these women than bed-rest.

Both women had uncomplicated vaginal deliveries with healthy babies. Case 1 was actually induced at 37 week and Case 2 (control) went into labor at 33 weeks GA. As shown by Maloni and colleagues, pregnancy outcomes differ depending on the degree of activity restriction [11]. For instance, women on complete bed rest had different pregnancy outcomes than those on partial bed rest. Thus, the occurrence of vaginal delivery of women on complete bed rest was lower than women on partial bed rest and with no bed rest and furthermore $65 \%$ of newborns were born prior to term [29].

Despite feeling more relaxed after the music intervention, Case 2 complained of swelling in the ankles and feet and also reported feeling tired, difficulty sleeping, no appetite and shortness of breath on exertion. Symptoms that are similar to those previously reported in the literature [11,12]. On the other hand, Case 1 reported less swelling in the lower extremities, more energy during the day and a better night's sleep. In addition, there were no significant changes in the frequency of uterine contractions due to exercise, which has also been reported previously $[25,26]$. Thus, a bed-rest-exercise program may be able to alleviate some of the side-effects of prolonged activity restriction in antenatal women with minimal risk.

Since the goal of the study was to determine feasibility of the bed-rest-exercise program, physiological measures such as weight, muscle atrophy or bone status were not measured but should be considered in future studies in order to better evaluate the side-effects of activity restriction and the possible counter effects of a bed-restexercise program.

The clinical setting can be a challenging area for this type of research. Researchers have to be prepared for last minute cancellations due to routine hospital care, unexpected visitors, or simply that patients are tired or simply preoccupied with other and certainly more important things on occasional days. Patients can also be released from hospital at any time depending on their condition and gestational age. Considering the importance of exercise in eliciting physiological responses, the effects of bed-rest-exercise needs further investigation. Pending these results, the chronic effects of a bed-restexercise program over long term to minimize physiological and psychosocial side-effects of prolonged bedrest in this population must also be assessed in larger randomized controlled studies.

\section{REFERENCES}

[1] Bloomfield, S.A. (1997) Changes in musculoskeletal structure and function with prolonged bed rest. Medicine \& Science in Sports \& Exercise, 29, 197-206. doi:10.1097/00005768-199702000-00006

[2] Convertino, V. and Hoffler, G.W. (1992) Cardiovascular physiology. Effects of microgravity. Journal of Florida Medical Association, 79, 517-524.

[3] Convertino, V.A. (1992) Effects of exercise and inactivity on intravascular volume and cardiovascular control mechanisms. Acta Astronautica, 27, 123-129. doi:10.1016/0094-5765(92)90188-O

[4] Convertino, V.A. (1997) Cardiovascular consequences of bed rest: Effect on maximal oxygen uptake. Medicine \& Science in Sports \& Exercise, 29, 191-196. doi:10.1097/00005768-199702000-00005

[5] Kasper, C.E, Talbot, L.A. and Gaines, J.M. (2002) Skeletal muscle damage and recovery. Advanced Practice in Acute \& Critical Care, 13, 237-247. doi:10.1097/00044067-200205000-00009

[6] Levine, B.D., Zuckerman, J.H. and Pawelczyk, J.A. (1997) Cardiac atrophy after bed-rest deconditioning: A nonneural mechanism for orthostatic intolerance. Circulation, 96, 517-525. doi:10.1161/01.CIR.96.2.517

[7] Vernikos, J., Ludwig, D.A., Ertl, A.C., Wade, C.E., Keil, L. and O'Hara, D. (1996) Effect of standing or walking on physiological changes induced by head down bed rest: Implications for spaceflight. Aviation, Space, and Environmental Medicine, 67, 1069-1079.

[8] Josten, L.E., Savik, K., Mullett, S.E., Campbell, R. and Vincent, P. (1995) Bedrest compliance for women with pregnancy problems. Birth, 22, 1-12.

[9] Saunders, M.C., Dick, J.S., Brown, I.M., McPherson, K. 
and Chalmers, I. (1985) The effects of hospital admission for bed rest on the duration of twin pregnancy: A randomised trial. Lancet, 2, 793-795. doi:10.1016/S0140-6736(85)90792-5

[10] Kovacevich, G.J., Gaich, S.A., Lavin, J.P., Hopkins, M.P., Crane, S.S., Stewart, J., et al. (2000) The prevalence of thromboembolic events among women with extended bed rest prescribed as part of the treatment for premature labor or preterm premature rupture of membranes. American Journal of Obstetrics \& Gynecology, 182, 10891092. doi:10.1067/mob.2000.105405

[11] Maloni, J.A., Chance, B., Zhang, C., Cohen, A.W., Betts, D. and Gange, S.J. (1993) Physical and psychosocial side effects of antepartum hospital bed rest. Nursing Research, 42, 197-203.

[12] Maloni, J.A. and Schneider, B.S. (2002) Inactivity: Symptoms associated with gastrocnemius muscle disuse during pregnancy. Advanced Practice in Acute \& Critical Care, 13, 248-262. doi:10.1097/00044067-200205000-00010

[13] Maloni, J.A., Alexander, G.R., Schluchter, M.D., Shah, D.M. and Park, S. (2004) Antepartum bed rest: Maternal weight change and infant birth weight. Biological Research for Nursing, 5, 177-186. doi:10.1177/1099800403260307

[14] Maloni, J.A., Margevicius, S.P. and Damato, E.G. (2006) Multiple gestation: Side effects of antepartum bed rest. Biological Research for Nursing, 8, 115-128. doi:10.1177/1099800406291455

[15] Maloni, J.A. (2010) Antepartum bed rest for pregnancy complications: Efficacy and safety for preventing preterm birth. Biological Research for Nursing, 12, 106-124. doi:10.1177/1099800410375978

[16] Maloni, J.A. and Park, S. (2005) Postpartum symptoms after antepartum bed rest. Journal of Obstetric, Gynecologic \& Neonatal Nursing, 34, 163-171. doi:10.1177/0884217504274416

[17] Glazener, C.M., Abdalla, M., Stroud, P., Naji, S., Templeton, A. and Russell, I.T. (1995) Postnatal maternal morbidity: Extent, causes, prevention and treatment. BJOG: An International Journal of Obstetrics \& Gynaecology, 102, 282-287. doi:10.1111/j.1471-0528.1995.tb09132.x

[18] Thompson, J.F., Roberts, C.L., Currie, M. and Ellwood, D.A. (2002) Prevalence and persistence of health problems after childbirth: Associations with parity and method of birth. Birth, 29, 83-94. doi:10.1046/j.1523-536X.2002.00167.X

[19] Lenz, E.R., Pugh, L.C., Milligan, R.A., Gift, A. and
Suppe, F. (1997) The middle-range theory of unpleasant symptoms: An update. ANS Advances in Nursing Science, 19, 14-27.

[20] Convertino, V.A., Bloomfield, S.A. and Greenleaf, J.E. (1997) An overview of the issues: physiological effects of bed rest and restricted physical activity. Medicine \& Science in Sports \& Exercise, 29, 187-190. doi:10.1097/00005768-199702000-00004

[21] Greenleaf, J.E. (1997) Intensive exercise training during bed rest attenuates deconditioning. Medicine \& Science in Sports \& Exercise, 29, 207-215. doi:10.1097/00005768-199702000-00007

[22] Ellis, S., Kirby, L.C. and Greenleaf, J.E. (1993) Lower extremity muscle thickness during 30-day 6 degrees headdown bed rest with isotonic and isokinetic exercise training. Aviation, Space, and Environtal Medicine, 64, 1011-1015.

[23] Akima, H., Kubo, K., Imai, M., Kanehisa, H., Suzuki, Y., Gunji, A., et al. (2001) Inactivity and muscle: Effect of resistance training during bed rest on muscle size in the lower limb. Acta Physiologica Scandinavica, 172, 269278. doi:10.1046/j.1365-201x.2001.00869.x

[24] Kawakami, Y., Akima, H., Kubo, K., Muraoka, Y., Hasegawa, H., Kouzaki, M., et al. (2001) Changes in muscle size, architecture, and neural activation after 20 days of bed rest with and without resistance exercise. European Journal of Applied Physiology, 84, 7-12. doi:10.1007/s004210000330

[25] Mayberry, L.J., Smith, M. and Gill, P. (1992) Effect of exercise on uterine activity in the patient in preterm labor. Journal of Perinatology, 12, 354-358.

[26] Brun, C.R., Shoemaker, J.K., Bocking, A., Hammond, J.A., Poole, M. and Mottola, M.F. (2011) Bed-rest exercise, activity restriction, and high-risk pregnancies: A feasibility study. Applied Physiology, Nutrition, and Metabolism, 36, 577-582. doi:10.1139/h11-036

[27] Maloni, J.A., Cohen, A.W. and Kane, J.H. (1998) Prescription of activity restriction to treat high-risk pregnancies. Journal of Women's Health, 7, 351-358. doi:10.1089/jwh.1998.7.351

[28] Maloni, J.A., Kane, J.H., Suen, L.J. and Wang, K.K. (2002) Dysphoria among high-risk pregnant hospitalized women on bed rest: A longitudinal study. Nursing Research, 51, 92-99. doi:10.1097/00006199-200203000-00005

[29] Maloni, J.A. (1993) Bed rest during pregnancy: Implications for nursing. Journal of Obstetric, Gynecology, Neonatal Nursing, 22, 422-426. doi:10.1111/j.1552-6909.1993.tb01825.x 